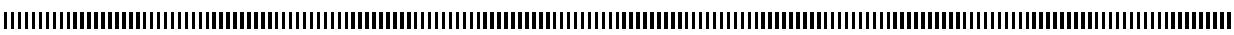

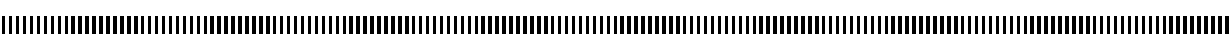

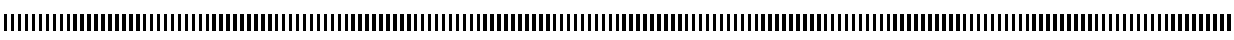

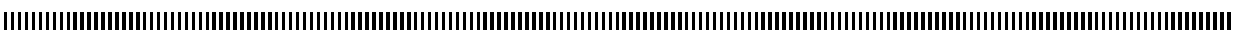

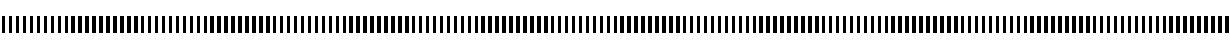

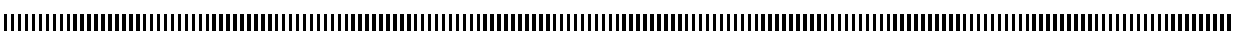

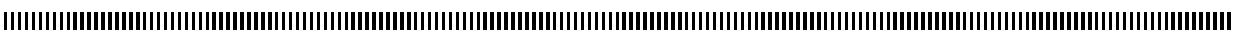

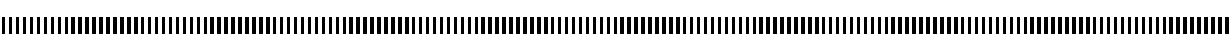

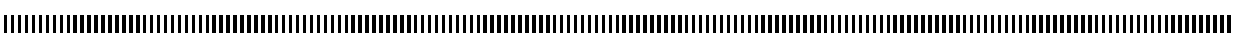

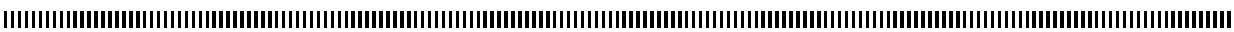

\title{
Comportement asymptotique d'un modèle à commutation de la prolifération du Typha
}

\author{
Mamadou Lamine Diagne a,c* - Papa Ibrahima Ndiaye b,a — Mary Teuw \\ Niane ${ }^{a}$ - Tewfik Sari c,d
}

a UMMISCO - Saint-Louis, Laboratoire d'Analyse Numérique et Informatique

Université Gaston Berger de Saint-Louis, BP 234 Sénégal

diagne12@yahoo.fr,mtniane@ugb.edu.sn

b Département de Maths, UFR SATIC, Université Alioune Diop, BP 30 Bambey, Sénégal

papaibra.ndiaye@uadb.edu.sn

c UHA, LMIA, Mulhouse, France

\& EPI Modemic Inria / Inra, UMR Mistea, SupAgro Montpellier, France

d Irstea, UMR Itap, Montpellier, France

Tewfik.Sari@irstea.fr

* Corresponding author.

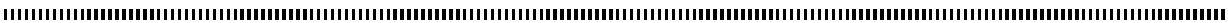
RÉSUMÉ. Dans cet article, nous proposons et étudions un modèle mathématique à commutation qui décrit la dynamique de population du Typha prenant en compte la saisonnalité de la reproduction sexuée de la plante. Ceci constitue une étape importante vers une meilleure compréhension de son developpement. L'étude théorique de ce modèle permet de déterminer les facteurs essentiels de la prolifération du Typha. Nous présentons d'abord l'analyse des solutions d'équilibre des sous-système qui composent le système à commutation ensuite nous étudions leur stabilité et enfin nous présentons une étude numérique du comportement asymptotique du système à commutation. Ceci constitue un premier pas vers une étude plus approfondie de la dynamique non linéaire de ce modèle.

ABSTRACT. In this paper we propose a mathematical model of the Typha growth and analyse its stability. The model that we plan to study describes the dynamics of the plant population. The theoretical study of this model determines the key factors of the Typha proliferation. We present the analysis of equilibrium solutions and lead a study of their local stability. This constitutes a first step towards a more detailed study of the nonlinear dynamics of this model.

MOTS-CLÉS : dynamique de croissance, stabilité, système non linéaire à commutation, Typha

KEYWORDS : dynamic growth, stability, switching system, Typha.

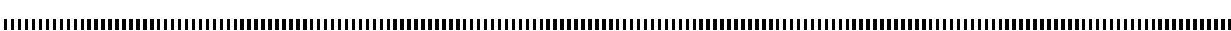

Received, March, 2011

Revised, Juin 25, 2012,

Revised, July 20, 2012

Accepted, August 22, 2012

Volume 15 - 2012, pages 101 à 122 - A R I M A

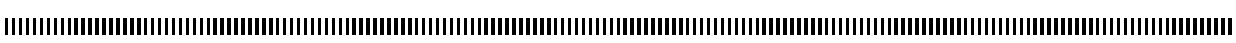

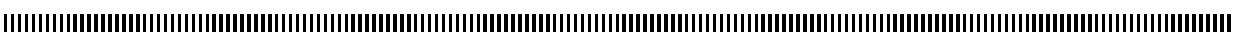

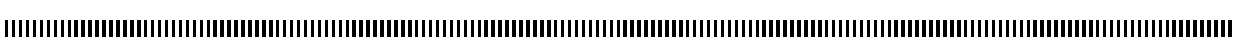

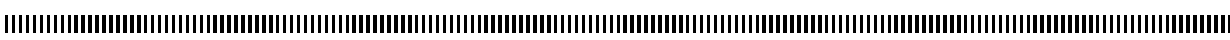

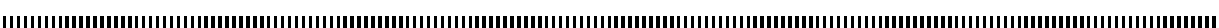




\section{Introduction}

Le Typha est une plante aquatique, invasive et pérenne. Il se développe grâce à deux modes de reproduction : une reproduction sexuée saisonnière et une multiplication végétative continue. Ces deux modes de reproduction ont donc lieu simultanément durant la période de la reproduction sexuée [2]. Ce mode de reproduction est entamé par la plante adulte avec la production de graines afin de donner naissance à des jeunes pousses. Quant à la multiplication végétative, dite aussi reproduction asexuée, elle démarre par la naissance de sortes de racines sur la plante adulte, appelées rhizomes [1]. Avec ces deux mécanismes de renouvellement, le genre Typha se développe dans les eaux peu profondes et peu saumâtres ou dans les parties d'un cours d'eau douce où le niveau de l'eau est inférieur à 3 mètres de profondeur, quelque soit le continent [5]. Cette étude s'intéresse au développement du Typha dans le Parc National des Oiseaux de Djoudj (PNOD) où la multiplication végétative débute entre 3 et 6 mois après la germination des graines et la reproduction sexuée se déroule entre les mois de mars et celui de juin d'une année donnée $[2]$.

Le PNOD est une zone humide située dans la vallée du fleuve sénégal. Il est aussi un lieu de rencontre d'oiseaux migrateurs paléatiques et afro-tropicaux [11], inscrit au patrimoine mondial de l'UNESCO en 1981. Le Typha y était présent vers les années 50. Mais, il avait disparu durant les années 70 à cause d'une longue période d'étiage et de sécheresse. A partir de la mise en service du barrage hydro-agricole de Diama en 1986, modifiant l'écosystème du bassin du fleuve sénégal, le Typha est réapparu dans cette zone. Dès lors, l'inquiétante invasion du Typha dans le PNOD a provoqué d'importantes nuisances environnementales, socio-économiques et sanitaires. Il s'agit essentiellement d'une perte de la biodiversité, d'une forte baisse de la rentabilité des activités économiques liées à l'inaccessibilité à l'eau pour l'agriculture, l'élevage et la pêche et l'occurence fréquente de certaines maladies telles que le paludisme et la bilharziose [10].

Les méthodes de lutte mécanique, biologique et chimique utilisées contre le Typha dans le PNOD ont eu une efficacité variable et limitée dans le temps. Ainsi, dans le cadre d'une étude pluridsiciplinaire pour mettre en place une stratégie de lutte écohydrologique, nous proposons un modèle mathématique à commutation décrivant la dynamique de population du Typha aux abords d'un point d'eau douce dans le Djoudj.

Dans ce travail, nous proposons l'analyse de la stabilité des points d'équilibre de chaque sous-système actif du modèle à commutation. Dans la Section 2, nous présentons le modèle à commutation et ses deux sous systèmes actifs. Dans la Section 3, nous définissons, pour chaque chacun des sous-système, un paramètre, appelé le taux de reproduction de base et nous démontrons que si ce paramètre est inférieur ou égal à 1 , le point d'équilibre nul est globalement asymptotiquement stable, et dans le cas contraire, si le taux de reproduction de base est supérieur à 1 , un point d'équilibre positif existe et 
est localement asymptotiquement stable. Dans la Section 4, nous présentons une étude numérique montrant l'existence d'un paramètre bifurcation de la durée de saisonnalité du modèle à commutation, avant de conclure dans la Section 5.

\section{Modèle}

Dans la modélisation de la dynamique de prolifération du Typha au voisinage d'un point d'eau prenant en compte la multiplication végétative et la saisonnalité de la reproduction sexuée, nous considérons trois compartiments : le compartiment des jeunes pousses provenant de la reproduction sexuée $\left(X_{1}\right)$, celui des jeunes pousses provenant de la reproduction asexuée $\left(X_{2}\right)$ et le compartiment des adultes $\left(X_{3}\right)$, i.e. les plantes matures capables de se reproduire suivant les deux modes. Soient $K$ la capacité de charge du milieu de développement du Typha et $Y(t)=X_{1}(t)+X_{2}(t)+X_{3}(t)$ le nombre total de plantes dans cet espace à l'instant $t$. Nous décrivons l'évolution de ces trois compartiments par le système d'équations différentielles ordinaires non linéaire et non autonome suivant :

$$
\left\{\begin{array}{l}
\dot{X}_{1}=\theta(t) X_{3}(1-Y / K)-\left(\gamma_{1}+\mu_{1}\right) X_{1} \\
\dot{X}_{2}=c_{2} X_{3}(1-Y / K)-\left(\gamma_{2}+\mu_{2}\right) X_{2} \\
\dot{X}_{3}=\gamma_{1} X_{1}+\gamma_{2} X_{2}-\mu_{3} X_{3}
\end{array}\right.
$$

où $\gamma_{1}$ (resp. $\gamma_{2}$ ) désigne le taux de passage de $X_{1}$ (resp. $X_{2}$ ) vers $X_{3}$. Les coefficients $\mu_{1}, \mu_{2}$ et $\mu_{3}$ désignent respectivement les taux de mortalité dans les compartiments $X_{1}$, $X_{2}$ et $X_{3}$. Le terme $(1-Y / K)$ désigne la probabilité de trouver de l'espace dans le milieu à l'instant $t$. Les paramètres $\theta(t)$ et $c_{2}$ désignent respectivement les taux de fécondité sans contrainte d'espace des adultes pour les jeunes pousses provenant de la reproduction sexuée et asexuée.

Tout au long de ce travail, nous supposons que le taux de fécondité saisonnière, $\theta(t)$ est une fonction périodique de période annuelle $(T=1$ an) définie par

$$
\theta(t)=\left\{\begin{array}{ll}
c_{1}, & \text { si } t \in[k T,(k+\alpha) T), \\
0, & \text { si } t \in[(k+\alpha) T,(k+1) T],
\end{array} \text { tel que } c_{1}>0\right.
$$

où $\alpha T, 0 \leq \alpha \leq 1$, est la fraction de l'année $k(k \in \mathbb{N})$ durant laquelle la reproduction sexuée s'effectue. Soient

$$
x_{1}=X_{1} / K, \quad x_{2}=X_{2} / K, \quad x_{3}=X_{3} / K, \quad y(t)=x_{1}(t)+x_{2}(t)+x_{3}(t) .
$$


Nous obtenons alors le modèle adimensionnel suivant :

$$
\left\{\begin{array}{l}
\dot{x_{1}}=\theta(t) x_{3}(1-y)-\left(\gamma_{1}+\mu_{1}\right) x_{1}, \\
\dot{x_{2}}=c_{2} x_{3}(1-y)-\left(\gamma_{2}+\mu_{2}\right) x_{2}, \\
\dot{x_{3}}=\gamma_{1} x_{1}+\gamma_{2} x_{2}-\mu_{3} x_{3} .
\end{array}\right.
$$

Comme la fonction $\theta(t)$ est une fonction $T$-périodique définie par (2.1), le système non linéaire et non autonome (2.2) devient un système à commutation avec les deux soussystèmes actifs suivants :

$$
\left\{\begin{array}{l}
\dot{x_{1}}=c_{1} x_{3}\left(1-x_{1}-x_{2}-x_{3}\right)-\left(\gamma_{1}+\mu_{1}\right) x_{1}, \\
\dot{x_{2}}=c_{2} x_{3}\left(1-x_{1}-x_{2}-x_{3}\right)-\left(\gamma_{2}+\mu_{2}\right) x_{2}, \\
\dot{x_{3}}=\gamma_{1} x_{1}+\gamma_{2} x_{2}-\mu_{3} x_{3} .
\end{array}\right.
$$

si $k T \leqslant t<(k+\alpha) T$, avec $k \in \mathbb{N}$ et

$$
\left\{\begin{array}{l}
\dot{x_{1}}=-\left(\gamma_{1}+\mu_{1}\right) x_{1}, \\
\dot{x_{2}}=c_{2} x_{3}\left(1-x_{1}-x_{2}-x_{3}\right)-\left(\gamma_{2}+\mu_{2}\right) x_{2}, \\
\dot{x_{3}}=\gamma_{1} x_{1}+\gamma_{2} x_{2}-\mu_{3} x_{3} .
\end{array}\right.
$$

si $(k+\alpha) T \leqslant t<(k+1) T$.

Nous étudions ces systèmes (2.2) (2.3) et (2.4) dans le domaine suivant :

$$
\Omega=\left\{\left(x_{1}, x_{2}, x_{3}\right) \in \mathbb{R}_{+}^{3}, \quad x_{1}+x_{2}+x_{3} \leq 1\right\} .
$$

Proposition 1. Le domaine $\Omega$ est positivement invariant pour le système (2.2).

Preuve. La positivité de la solution est garantie par le fait que si on annule une variable, sa dérivée reste positive. On évalue donc $\dot{x}_{1}, \dot{x}_{2}$ et $\dot{x}_{3}$ en $x_{1}=0, x_{2}=0$ et $x_{3}=0$. On obtient alors

$$
\begin{gathered}
x_{1}=0 \text { et } x_{2}+x_{3} \leq 1 \Longrightarrow \dot{x}_{1}=c_{1} x_{3}\left(1-x_{2}-x_{3}\right) \geq 0, \\
x_{2}=0 \text { et } x_{1}+x_{3} \leq 1 \Longrightarrow \dot{x}_{2}=c_{2} x_{3}\left(1-x_{1}-x_{3}\right) \geq 0, \\
x_{3}=0 \text { et } x_{1}+x_{2} \leq 1 \Longrightarrow \dot{x}_{3}=\gamma_{1} x_{1}+\gamma_{2} x_{2} \geq 0 .
\end{gathered}
$$

Maintenant, il reste à montrer que la variable $y(t)$ reste inférieure à 1 . On a

$$
\dot{y}=x_{3}\left(c_{2}+\theta(t)\right)(1-y)-\mu_{1} x_{1}-\mu_{2} x_{2}-\mu_{3} x_{3} .
$$

On en déduit donc,

$$
y=1 \text { et } x_{1} \geq 0, x_{2} \geq 0, x_{3} \geq 0 \Rightarrow \dot{y}=-\mu_{1} x_{1}-\mu_{2} x_{2}-\mu_{3} x_{3} \leq 0 .
$$

Ce qui permet de conclure que, pour toute condition initiale dans $\Omega$, la frontière de $\Omega$ n'est pas franchissable.

\section{A R I M A}




\section{Points d'équilibre des sous-systèmes et analyse de leur stabilité}

Avant d'étudier de la stabilité asymptotique du système à commutation (2.2), nous abordons dans cette section l'étude de la stabilité asymptotique des points d'équilibre de chaque sous-système (2.3) et (2.4).

Dans le calcul des points d'équilibre du sous-système (2.3), voir Section A, nous avons obtenu le paramètre :

$$
R_{0}=R_{0,1}+R_{0,2}
$$

où

$$
R_{0,1}=\frac{c_{1} \gamma_{1}}{\mu_{3}\left(\gamma_{1}+\mu_{1}\right)} \text { et } R_{0,2}=\frac{c_{2} \gamma_{2}}{\mu_{3}\left(\gamma_{2}+\mu_{2}\right)} .
$$

Ce paramètre $R_{0}$ représente le taux de reproduction de base de la plante adulte associé à la fois à la reproduction sexuée et à la mutiplication végétative. De même, le paramètre $R_{0,2}$ représente le taux de reproduction de base de la plante adulte associé à la multiplication végétative qui est seulement pris en compte lorsque $\theta(t)=0$ (sous-système (2.4)). De la relation $R_{0}=R_{0,1}+R_{0,2}$ et de l'expression de $R_{0,1}$, ce dernier paramètre désigne le taux de reproduction de base de la plante adulte associé à la reproduction sexuée.

Si $R_{0} \leq 1$, le sous-système (2.3) admet l'unique point d'équilibre $E_{0}=(0,0,0)$. Si $R_{0}>1$, il admet un second point d'équilibre $E_{1}$ donné par

$$
E_{1}=\left(\begin{array}{l}
\frac{c_{1} \mu_{3}\left(\gamma_{2}+\mu_{2}\right)}{c_{1}\left(\gamma_{1}+\mu_{3}\right)\left(\gamma_{2}+\mu_{2}\right)+c_{2}\left(\gamma_{2}+\mu_{3}\right)\left(\gamma_{1}+\mu_{1}\right)} \frac{\left(R_{0}-1\right)}{R_{0}} \\
\frac{c_{2} \mu_{3}\left(\gamma_{1}+\mu_{1}\right)}{c_{1}\left(\gamma_{1}+\mu_{3}\right)\left(\gamma_{2}+\mu_{2}\right)+c_{2}\left(\gamma_{2}+\mu_{3}\right)\left(\gamma_{1}+\mu_{1}\right)} \frac{\left(R_{0}-1\right)}{R_{0}} \\
\frac{\mu_{3}\left(\gamma_{1}+\mu_{1}\right)\left(\gamma_{2}+\mu_{2}\right)}{c_{1}\left(\gamma_{1}+\mu_{3}\right)\left(\gamma_{2}+\mu_{2}\right)+c_{2}\left(\gamma_{2}+\mu_{3}\right)\left(\gamma_{1}+\mu_{1}\right)}\left(R_{0}-1\right)
\end{array}\right)
$$

De la même manière, si $R_{0,2} \leq 1$ alors le sous-système (2.4) admet l'unique point d'équilibre $E_{0}=(0,0,0)$. Sinon, lorsque $R_{0,2}>1$, ce sous-système admet un second point d'équilibre $E_{2}$ donné par

$$
E_{2}=\left(\begin{array}{c}
0 \\
\frac{\mu_{3}}{\left(\gamma_{2}+\mu_{3}\right)} \frac{\left(R_{0,2}-1\right)}{R_{0,2}} \\
\frac{\mu_{3}\left(\gamma_{2}+\mu_{2}\right)}{c_{2}\left(\gamma_{2}+\mu_{3}\right)}\left(R_{0,2}-1\right)
\end{array}\right)
$$


Il faut noter que le système (2.4) s'obtient tout simplement en posant $c_{1}=0$ dans le système (2.3). On vérifie que si on pose $c_{1}=0$ dans (3.3) on obtient bien (3.4). Ainsi, la multiciplicité des points d'équilibres de chaque sous système dépend de la valeur d'un paramètre de bifurcation qui n'est rien d'autre que le taux de reproduction de base $R_{0}$ ou $R_{0,2}$ associé au sous-système considéré.

L'analyse de la stabilité des points d'équilibre du sous-système (2.3) en fonction de la valeur du paramètre $R_{0}$ est résumée dans la proposition suivante.

Proposition 2. Si $R_{0} \leqslant 1, E_{0}=(0,0,0)$ est globalement asymptotiquement stable (GAS) pour le sous-système (2.3). Si $R_{0}>1$, l'équilibre $E_{0}$ est instable et l'équilibre $E_{1}$ est localement asymptotiquement stable (LAS) pour le sous-système (2.3).

Preuve. Nous partageons la preuve en deux parties suivant que $R_{0} \leq 1$ ou $R_{0}>1$.

Supposons que $R_{0} \leqslant 1$. Montrons que $E_{0}$ est globalement asymptotiquement stable. Soit la fonction

$$
V: \mathbb{R}_{+}^{3} \longrightarrow \mathbb{R}
$$

définie par

$$
V\left(x_{1}, x_{2}, x_{3}\right)=\gamma_{1}\left(\gamma_{2}+\mu_{2}\right) x_{1}+\gamma_{2}\left(\gamma_{1}+\mu_{1}\right) x_{2}+\left(\gamma_{1}+\mu_{1}\right)\left(\gamma_{2}+\mu_{2}\right) x_{3}
$$

On a

$$
V\left(E_{0}\right)=0
$$

et pour tout $\left(x_{1}, x_{2}, x_{3}\right) \in \Omega \backslash\left\{E_{0}\right\}, V\left(x_{1}, x_{2}, x_{3}\right)>0$.

Par ailleurs, la dérivée de $V$ le long des trajectoires de (2.2) est

$$
\begin{aligned}
\dot{V}= & \gamma_{1}\left(\gamma_{2}+\mu_{2}\right) \dot{x}_{1}+\gamma_{2}\left(\gamma_{1}+\mu_{1}\right) \dot{x}_{2}+\left(\gamma_{1}+\mu_{1}\right)\left(\gamma_{2}+\mu_{2}\right) \dot{x}_{3} \\
= & c_{1} \gamma_{1}\left(\gamma_{2}+\mu_{2}\right)\left(1-x_{1}-x_{2}-x_{3}\right) x_{3}-\left(\gamma_{2}+\mu_{2}\right)\left(\gamma_{1}+\mu_{1}\right) \gamma_{1} x_{1} \\
& +c_{2} \gamma_{2}\left(\gamma_{1}+\mu_{1}\right)\left(1-x_{1}-x_{2}-x_{3}\right) x_{3}-\left(\gamma_{1}+\mu_{1}\right)\left(\gamma_{2}+\mu_{2}\right) \gamma_{2} x_{2} \\
& +\left(\gamma_{1}+\mu_{1}\right)\left(\gamma_{2}+\mu_{2}\right) \gamma_{1} x_{1}+\left(\gamma_{1}+\mu_{1}\right)\left(\gamma_{2}+\mu_{2}\right) \gamma_{2} x_{2} \\
& -\left(\gamma_{1}+\mu_{1}\right)\left(\gamma_{2}+\mu_{2}\right) \mu_{3} x_{3} \\
= & x_{3}\left[c_{1} \gamma_{1}\left(\gamma_{2}+\mu_{2}\right)+c_{2} \gamma_{2}\left(\gamma_{1}+\mu_{1}\right)-\mu_{3}\left(\gamma_{1}+\mu_{1}\right)\left(\gamma_{2}+\mu_{2}\right)\right] \\
& -x_{3}\left(x_{1}+x_{2}+x_{3}\right)\left[c_{1} \gamma_{1}\left(\gamma_{2}+\mu_{2}\right)+c_{2} \gamma_{2}\left(\gamma_{1}+\mu_{1}\right)\right] \\
= & -x_{3}\left(x_{1}+x_{2}+x_{3}\right)\left[c_{1} \gamma_{1}\left(\gamma_{2}+\mu_{2}\right)+c_{2} \gamma_{2}\left(\gamma_{1}+\mu_{1}\right)\right] \\
& x_{3} \mu_{3}\left(\gamma_{1}+\mu_{1}\right)\left(\gamma_{2}+\mu_{2}\right)\left(R_{0}-1\right) .
\end{aligned}
$$

Comme $R_{0} \leqslant 1$, on a $\dot{V} \leq 0$ dans $\Omega$.

Ainsi, $V$ est une fonction de Lyapunov et la dérivée de la fonction de Lyapunov le long des trajectoires du sous-système est semi-définie négative.

La partie $S \subset \Omega$ tel que ses éléments vérifient $\dot{V}=0$ le long des trajectoires est le suivant

$$
S=\left\{\left(x_{1}, x_{2}, x_{3}\right) \in \Omega: x_{3}=0\right\} .
$$


Nous vérifions aisément que toute trajectoire du sous-système contenue dans $S$ est telle que $x_{3}(t)$ est identiquement nulle. Ceci implique immédiatement que $\dot{x}_{3}(t)$ est identiquement nulle. Donc, la troisième équation du sous-système (2.3) devient $\gamma_{1} x_{1}+\gamma_{2} x_{2}=0$. Comme les variables et les paramètres du sous-système (2.3) sont positifs, nous obtenons $x_{1}=x_{2}=0$. Donc, la seule trajectoire contenue dans $S$ est celle de l'équilibre. D'où, d'après le principe d'invariance de LaSalle [7], l'équilibre $E_{0}$ est gobalement asymptotiquement stable pour le sous-système (2.3).

Supposons maintenant que $R_{0}>1$.

- Montrons que $E_{0}$ est instable.

La matrice jacobienne du sous-système (2.3) au point $E_{0}$ est donnée par

$$
J\left(E_{0}\right)=\left(\begin{array}{ccc}
-\left(\gamma_{1}+\mu_{1}\right) & 0 & c_{1} \\
0 & -\left(\gamma_{2}+\mu_{2}\right) & c_{2} \\
\gamma_{1} & \gamma_{2} & -\mu_{3}
\end{array}\right)
$$

Son polynôme caractéristique $P$, obtenu avec le logiciel de calcul formel Maxima, est donné par

$$
-P(X)=X^{3}+a_{1} X^{2}+a_{2} X+a_{3}
$$

avec

$$
\begin{aligned}
a_{1}= & \gamma_{2}+\mu_{2}+\gamma_{1}+\mu_{1}+\mu_{3}>0 \\
a_{2}= & -c_{1} \gamma_{1}-c_{2} \gamma_{2}+\gamma_{1} \gamma_{2}+\gamma_{1} \mu_{2}+\gamma_{1} \gamma_{3}+\gamma_{2} \mu_{1}+\gamma_{2} \gamma_{3}+\mu_{1} \mu 2+\mu_{1} \mu 3 \\
& +\mu_{2} \mu_{3} \\
a_{3}= & \mu_{1} \mu_{2} \mu_{3}+\gamma_{1} \mu_{2} \mu_{3}+\gamma_{2} \mu_{1} \mu_{3}+\gamma_{1} \gamma_{2} \mu_{3}-c_{1} \gamma_{1} \mu_{2}-c_{2} \gamma_{2} \mu_{1}-c_{2} \gamma_{1} \gamma_{2} \\
& -c_{1} \gamma_{1} \gamma_{2} .
\end{aligned}
$$

Il est clair que le coefficient $a_{3}$ peut s'écrire

$$
\begin{aligned}
a_{3} & =\mu_{3}\left(\mu_{1}+\gamma_{1}\right)\left(\gamma_{2}+\mu_{2}\right)-c_{1} \gamma_{1}\left(\gamma_{2}+\mu_{2}\right)-c_{2} \gamma_{2}\left(\gamma_{1}+\mu_{1}\right) \\
& =\mu_{3}\left(\mu_{1}+\gamma_{1}\right)\left(\mu_{2}+\gamma_{2}\right)\left(1-R_{0}\right)
\end{aligned}
$$

Comme $R_{0}>1$ alors $a_{3}<0$, donc, $a_{1}$ et $a_{3}$ sont de signes opposés. Par consequent, d'après le critère de Routh-Hurwitz [4] la matrice $J\left(E_{0}\right)$ a une valeur propre à partie réelle positive. Donc, l'équilibre $E_{0}$ est instable.

- Montrons que $E_{1}$ est localement asymptotiquement stable.

La matrice jacobienne du sous-système (2.3) au point $E_{1}$ est donnée par

$$
J\left(E_{1}\right)=\left(\begin{array}{ccc}
-c_{1} x_{3}^{*}-\left(\gamma_{1}+\mu_{1}\right) & -c_{1} x_{3}^{*} & c_{1}\left(1-x_{1}^{*}-x_{2}^{*}-2 x_{3}^{*}\right) \\
-c_{2} x_{3}^{*} & -c_{2} x_{3}^{*}-\left(\gamma_{2}+\mu_{2}\right) & c_{2}\left(1-x_{1}^{*}-x_{2}^{*}-2 x_{3}^{*}\right) \\
\gamma_{1} & \gamma_{2} & -\mu_{3}
\end{array}\right) .
$$


Son polynôme caractéristique, obtenu avec Maxima est

$$
-P(X)=X^{3}+b_{1} X^{2}+b_{2} X+b_{3} .
$$

avec

$$
b_{1}=c_{1} x_{3}^{*}+c_{2} x_{3}^{*}+\gamma_{1}+\gamma_{2}+\mu_{1}+\mu_{2}+\mu_{3}>0
$$

et

$$
\begin{aligned}
b_{2}= & c_{2} \mu_{3} x_{3}^{*}+c_{1} \mu_{3} x_{3}^{*}+c_{1} \mu_{2} x_{3}^{*}+c_{2} \mu_{1} x_{3}^{*}+2 c_{2} \gamma_{2} x_{3}^{*}+c_{1} \gamma_{1} x_{3}^{*}+c_{2} \gamma_{1} x_{3}^{*} \\
& +2 c_{1} \gamma_{1} x_{3}^{*}+c_{2} \gamma_{2} x_{2}^{*}+c_{1} \gamma_{1} x_{2}^{*}+c_{2} \gamma_{2} x_{1}^{*}+c_{1} \gamma_{1} x_{1}^{*}+\mu_{2} \mu_{3}+\mu_{1} \mu_{3} \\
& +\gamma_{2} \mu_{3}+\gamma_{1} \mu_{3}+\mu_{1} \mu_{2}+\gamma_{1} \mu_{2}+\gamma_{2} \mu_{1}+\gamma_{1} \gamma_{2}-c_{2} \gamma_{2}-c_{1} \gamma_{1} \\
b_{3}= & c_{1} \mu_{2} \mu_{3} x_{3}^{*}+c_{2} \mu_{1} \mu_{3} x_{3}^{*}+c_{1} \gamma_{2} \mu_{3} x_{3}^{*}+c_{2} \gamma_{1} \mu_{3} x_{3}^{*}+2 c_{1} \gamma_{1} \mu_{2} x_{3}^{*} \\
& +2 c_{2} \gamma_{2} \mu_{1} x_{3}^{*}+2 c_{2} \gamma_{1} \gamma_{2} x_{3}^{*}+2 c_{1} \gamma_{1} \gamma_{2} x_{3}^{*}+c_{1} \gamma_{1} \mu_{2} x_{2}^{*}+c_{2} \gamma_{2} \mu_{1} x_{2}^{*} \\
& +c_{2} \gamma_{1} \gamma_{2} x_{2}^{*}+c_{1} \gamma_{1} \gamma_{2} x_{2}^{*}+c_{1} \gamma_{1} \mu_{2} x_{1}^{*}+c_{2} \gamma_{2} \mu_{1} x_{1}^{*}+c_{2} \gamma_{1} \gamma_{2} x_{1}^{*} \\
& +c_{1} \gamma_{1} \gamma_{2} x_{1}^{*}+\mu_{1} \mu_{2} \mu_{3}+\gamma_{1} \mu_{2} \mu_{3}+\gamma_{2} \mu_{1} \mu_{3}+\gamma_{1} \gamma_{2} \mu_{3}-c_{1} \gamma_{1} \mu_{2} \\
& -c_{2} \gamma_{2} \mu_{1}-c_{2} \gamma_{1} \gamma_{2}-c_{1} \gamma_{1} \gamma_{2} .
\end{aligned}
$$

On réécrit $b_{2}$ sous la forme

$$
\begin{aligned}
b_{2}= & c_{1} \gamma_{1}\left(x_{1}^{*}+x_{2}^{*}+x_{3}^{*}-1\right)+c_{2} \gamma_{2}\left(x_{1}^{*}+x_{2}^{*}+x_{3}^{*}-1\right)+\left[c_{1}\left(\gamma_{1}+\mu_{3}\right)\right. \\
& \left.+c_{2}\left(\gamma_{2}+\mu_{3}\right)+c_{1}\left(\mu_{2}+\gamma_{2}\right)+c_{2}\left(\mu_{1}+\gamma_{2}\right)\right] x_{3}^{*}+\left(\gamma_{2}+\mu_{2}\right) \mu_{3} \\
& +\left(\gamma_{1}+\mu_{1}\right) \mu_{3}+\left(\gamma_{1}+\mu_{1}\right)\left(\gamma_{2}+\mu_{2}\right) .
\end{aligned}
$$

Or

$$
x_{1}^{*}+x_{2}^{*}+x_{3}^{*}=1-\frac{1}{R_{0}} .
$$

En utilisant (3.6), nous avons

$$
\begin{aligned}
b_{2}= & +\left[c_{1}\left(\gamma_{1}+\mu_{3}\right)+c_{2}\left(\gamma_{2}+\mu_{3}\right)+c_{1}\left(\mu_{2}+\gamma_{2}\right)+c_{2}\left(\mu_{1}+\gamma_{2}\right)\right] x_{3}^{*} \\
& -c_{1} \gamma_{1} \frac{1}{R_{0}}-c_{2} \gamma_{2} \frac{1}{R_{0}}+\left(\gamma_{2}+\mu_{2}\right) \mu_{3}+\left(\gamma_{1}+\mu_{1}\right) \mu_{3} \\
& +\left(\gamma_{1}+\mu_{1}\right)\left(\gamma_{2}+\mu_{2}\right) .
\end{aligned}
$$


Par consï $; \frac{1}{2}$ quent

$$
\begin{aligned}
b_{2}= & {\left[c_{1}\left(\gamma_{1}+\mu_{3}\right)+c_{2}\left(\gamma_{2}+\mu_{3}\right)+c_{1}\left(\mu_{2}+\gamma_{2}\right)+c_{2}\left(\mu_{1}+\gamma_{2}\right)\right] x_{3}^{*} } \\
& +\left(\gamma_{1}+\mu_{1}\right)\left(\gamma_{2}+\mu_{2}\right)+\mu_{3}\left(\gamma_{1}+\mu_{1}\right)\left(1-\frac{R_{0,1}}{R_{0}}\right) \\
& +\mu_{3}\left(\gamma_{2}+\mu_{2}\right)\left(1-\frac{R_{0,2}}{R_{0}}\right)
\end{aligned}
$$

Or $R_{0}=R_{0,1}+R_{0,2}$, donc, nous avons $b_{2}>0$.

On réécrit $b_{3}$ sous la forme

$$
\begin{aligned}
b_{3}= & c_{1} \gamma_{1} \gamma_{2}\left(x_{1}^{*}+x_{2}^{*}+x_{3}^{*}-1\right)+c_{1} \gamma_{1} \mu_{2}\left(x_{1}^{*}+x_{2}^{*}+x_{3}^{*}-1\right) \\
& +c_{2} \gamma_{2} \gamma_{1}\left(x_{1}^{*}+x_{2}^{*}+x_{3}^{*}-1\right)+c_{2} \gamma_{2} \mu_{1}\left(x_{1}^{*}+x_{2}^{*}+x_{3}^{*}-1\right) \\
& +c_{1} \gamma_{1} \gamma_{2} x_{3}^{*}+c_{1} \gamma_{1} \mu_{2} x_{3}^{*}+c_{2} \gamma_{2} \gamma_{1} x_{3}^{*}+c_{2} \gamma_{2} \mu_{1} x_{3}^{*}+c_{1} \mu_{2} \mu_{3} x_{3}^{*} \\
& +c_{2} \mu_{1} \mu_{3} x_{3}^{*}+c_{1} \gamma_{2} \mu_{3} x_{3}^{*}+c_{2} \gamma_{1} \mu_{3} x_{3}^{*}+\mu_{1} \mu_{2} \mu_{3}+\gamma_{1} \mu_{2} \mu_{3} \\
& +\gamma_{2} \mu_{1} \mu_{3}+\gamma_{1} \gamma_{2} \mu_{3}
\end{aligned}
$$

En utilisant (3.6), on obtient

$$
\begin{aligned}
b_{3}= & -\frac{1}{R_{0}}\left[c_{1} \gamma_{1}\left(\gamma_{2}+\mu_{2}\right)+c_{2} \gamma_{2}\left(\gamma_{1}+\mu_{1}\right)\right]+\left[c_{1}\left(\gamma_{1}+\mu_{3}\right)\left(\mu_{2}+\gamma_{2}\right)\right. \\
& \left.+c_{2}\left(\gamma_{2}+\mu_{3}\right)\left(\mu_{1}+\gamma_{2}\right)\right] x_{3}^{*}+\mu_{3}\left(\gamma_{1}+\mu_{1}\right)\left(\gamma_{2}+\mu_{2}\right)
\end{aligned}
$$

On déduit $b_{3}$ en utilisant l'expression de $R_{0}$ dans l'équation (3.2)

$$
b_{3}=\left[c_{1}\left(\gamma_{1}+\mu_{3}\right)\left(\mu_{2}+\gamma_{2}\right)+c_{2}\left(\gamma_{2}+\mu_{3}\right)\left(\mu_{1}+\gamma_{2}\right)\right] x_{3}^{*}>0
$$

En utilisant les les expressions (3.5) et (3.7) de $b_{1}$ et $b_{2}$ on obtient

$$
b_{1} b_{2}=\left[c_{1}\left(\gamma_{1}+\mu_{3}\right)\left(\mu_{2}+\gamma_{2}\right)+c_{2}\left(\gamma_{2}+\mu_{3}\right)\left(\mu_{1}+\gamma_{2}\right)\right] x_{3}^{*}+Q=b_{3}+Q
$$

avec $Q>0$. Par conséquent, nous avons $b_{1}>0, b_{2}>0, b_{3}>0$ et $\left(b_{1} b_{2}-b_{3}\right)>0$. D'aprés le critére de Routh-Hurwitz, on en déduit que les valeurs propres de la matrice jacobienne $J\left(E_{1}\right)$ sont de parties réelles strictement négatives. Donc, l'équilibre $E_{1}$ est asymptotiquement stable si $R_{0}>1$.

La stabilité des points d'équilibre du sous-système (2.4) dépend du paramètre $R_{0,2}$. Ce résultat est exposé dans le corollaire suivant de la Proposition 2 .

Corollaire 1. Si $R_{0,2} \leqslant 1, E_{0}$ est globalement asymptotiquement stable dans le domaine $\Omega$. Si $R_{0,2}>1$, l'équilibre $E_{0}$ est instable et l'équilibre $E_{2}$ est localement asymptotiquement stable. 
La stabilité asymptotique de chacun des sous-systèmes ne suffit pas à conclure la stabilité asymptotique uniforme du système à commutation (2.2).

\section{Résultats de simulations numériques}

Dans cette section, nous illustrons par quelques simulations numériques le comportement asymptotique du système à commutation (2.2) en fonction de la stabilité ou de l'instabilité de ses sous-systèmes. Ici, nous considèrons les paramètres seuils suivants :

$$
R_{0}(t)=R_{0,1}(t)+R_{0,2}, \text { où } R_{0,1}(t)=\frac{\theta(t) \gamma_{1}}{\mu_{3}\left(\gamma_{1}+\mu_{1}\right)} \quad \text { et } \quad R_{0,2}=\frac{c_{2} \gamma_{2}}{\mu_{3}\left(\gamma_{2}+\mu_{2}\right)} \text {. }
$$

Ainsi,

$$
R_{0}(t)=\left\{\begin{aligned}
R_{0}, & \text { si } t \in[k T,(k+\alpha) T[ \\
R_{0,2}, & \text { si } t \in[(k+\alpha),(k+1) T[
\end{aligned}\right.
$$

Dans toutes les simulations numériques suivantes, nous considèrons les valeurs des paramètres définies dans le tableau 1.

\begin{tabular}{|c|c|c|c|}
\hline Définitions & Symboles & Valeurs & Unités \\
\hline Taux de passage de $x_{1}$ vers $x_{3}$ & $\gamma_{1}$ & $1 / 8$ & mois $^{-1}$ \\
\hline Taux de passage de $x_{2}$ vers $x_{3}$ & $\gamma_{2}$ & $1 / 6$ & mois $^{-1}$ \\
\hline Durée de vie moyenne des $x_{1}$ & $1 / \mu_{1}$ & 24 & mois \\
\hline Durée de vie moyenne des $x_{2}$ & $1 / \mu_{2}$ & 24 & mois \\
\hline Durée de vie moyenne des $x_{3}$ & $1 / \mu_{3}$ & 72 & mois \\
\hline
\end{tabular}

Tableau 1 - Définitions et valeurs des paramètres pour les simulations.

Théoriquement, lorsque $R_{0} \leq 1$, le point d'équilibre $E_{0}$ est GAS pour le soussystème (2.3) (Proposition 2) d'une part et d'autre part pour le sous-système (2.4) (Corollaire 1). Dans ce cas, nous avons $R_{0}(t) \leq 1, \forall t \geq 0$ et les simulations numériques du système à commutation (2.2) composé des deux sous-systèmes (2.3) et (2.4) présentés dans la figure 1 montrent que celui-ci converge vers $E_{0}$ suivant certaines valeurs des paramètres (voir figure 1).

\section{A R I M A}



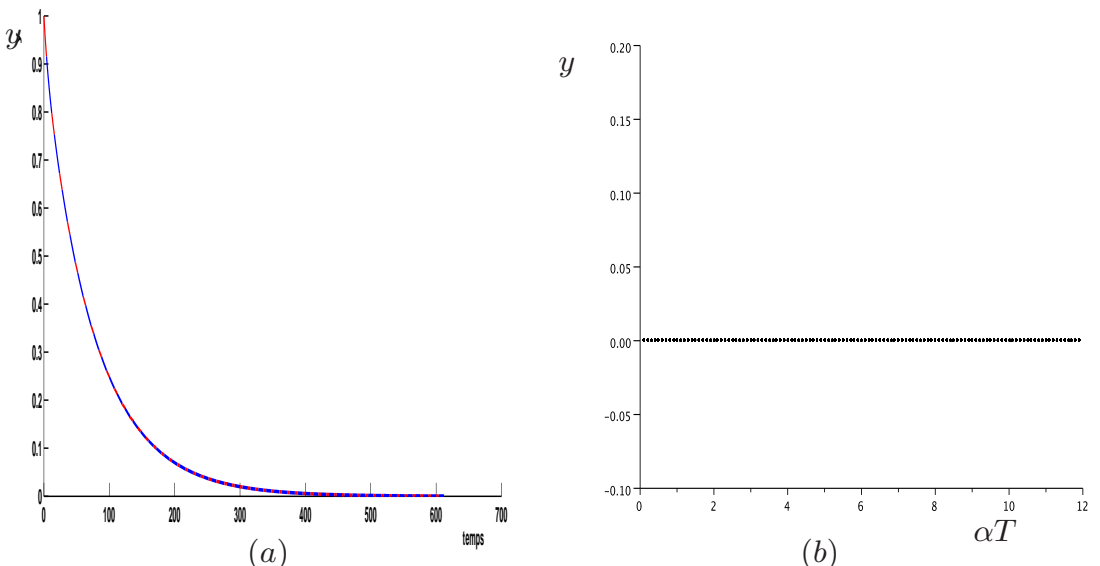

(b)

Figure 1 - (a) Évolution au cours temps de la population totale $y=x_{1}+x_{2}+x_{3}$ du système à commutation (2.2) pour $\alpha=1 / 4$ avec la condition initiale $Y_{0}=(0.3,0.1,0.6)$. (b) Courbe des valeurs asymptotiques de la population totale $y$ du système à commutation (2.2) en fonction de $\alpha T(0 \leq \alpha \leq 1)$. Pour toute valeur de $\alpha T$, les 120 dernières valeurs de $y$ sont représentées lorsque les valeurs des paramètres sont : $c_{1}=0.002, c_{2}=0.001$, $T=12$ et dans le tableau 1 . Dans ce cas, $R_{0,1}=0.1080, R_{0,2}=0.0576<1$ et $R_{0}(t)=R_{0,1}(t)+R_{0,2}<1$ et le système à commutation converge vers $E_{0}$.

En effet, d'une part la population totale $y=x_{1}+x_{2}+x_{3}$ tend vers 0 lorsque $t$ tend vers $+\infty$ et la durée de la reproduction sexuée $\alpha=1 / 4$ (voir figure 1 (a)). Et d'autre part, les valeurs asymptotiques de la population totale $y=x_{1}+x_{2}+x_{3}$ sont toutes nulles quelque soit la durée de la période de la reproduction sexuée $\alpha$ (voir figure 1 (b)). Ce résultat de simulations numériques suggère que, théoriquement, le système à commutation (2.2) converge asymptotiquement vers $E_{0}$ pour certaines valeurs des paramètres telles que $R_{0}(t)<1$.

Dans le cas où $R_{0}>1$, nous avons montré théoriquement que $E_{0}$ est instable et $E_{1}$ est LAS pour le système (2.3). Mais, pour le sous-système (2.3) les trajectoires issues des quatre conditions initiales $I_{1}=(0.9,0.01,0.01), I_{2}=(0.01,0.9,0.01), I_{3}=$ $(0.01,0.01,0.01)$ et $I_{4}=(0.1,0.2,0.5)$ se trouvant aux coins du simplexe $\Omega$ et loin du voisinage de l'équilibre positive $E_{1}$ convergent toutes vers ce point lorsque $R_{0}>1$ pour les valeurs numériques testées selon les cas : $R_{0,1}<1$ et $R_{0,2}>1$ (voir figure 2), $R_{0,1}>1$ et $R_{0,2}<1$ (voir figure 3), $R_{0,1}>1, R_{0,2}>1$ (voir figure 4) et $R_{0,1}<1$ et $R_{0,2}<1$ (voir figure 5 ). 

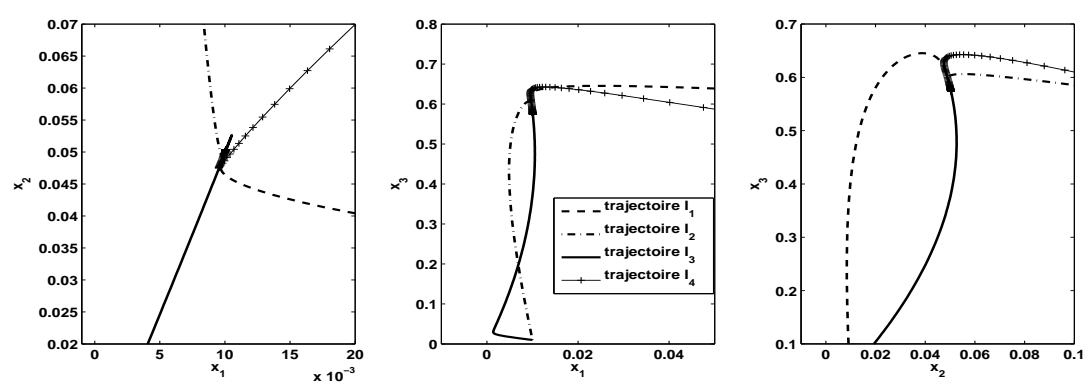

Figure 2 - Projections du portrait de phase du sous-système autonome (2.3) dans les plans $\left(x_{1}, x_{2}\right),\left(x_{1}, x_{2}\right)$ et $\left(x_{2}, x_{3}\right)$ lorsque $R_{0,1}<1, R_{0,2}>1$ et $R_{0}>1$. Nous illustrons la convergence du sous-système (2.3) vers l'équilibre $E_{1}=(0.0101,0.0504,0.5741)$ lorsque $c_{1}=0.01, c_{2}=0.04$ et toutes les valeurs des autres paramètres sont dans le tableau 1 . Avec ces valeurs, nous avons $R_{0,1}=0.576, R_{0,2}=2.1600$ et $R_{0}=2.7360$.
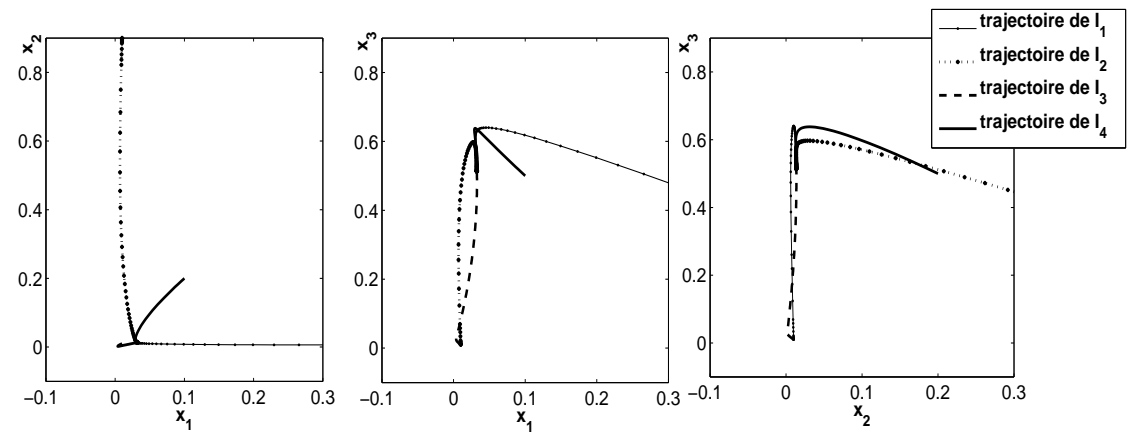

Figure 3 - Projections du portrait de phase du sous-système autonome (2.3) dans les plans $\left(x_{1}, x_{2}\right),\left(x_{1}, x_{2}\right)$ et $\left(x_{2}, x_{3}\right)$ lorsque $R_{0}>1$. Nous illustrons la convergence $\mathrm{du}$ sous-système (2.3) vers l'équilibre $E_{1}=(0.0404,0.0108,0.4934)$, lorsque $c_{1}=0.03$, $c_{2}=0.01$ et toutes les valeurs des autres paramètres sont dans le tableau 1 . Dans ce cas, nous avons $R_{0}=2.196>1, R_{0,1}=1,620$ et $R_{0,2}=0.576$. 

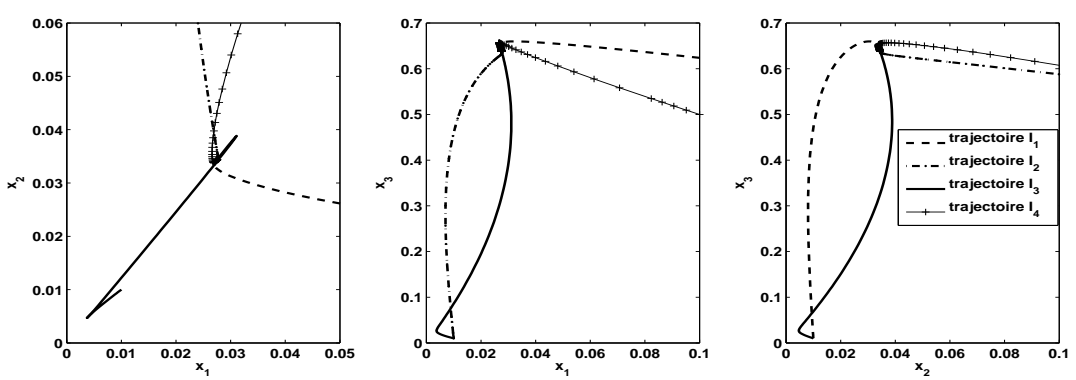

Figure 4 - Projections du portrait de phase du sous-système autonome (2.3) dans les plans $\left(x_{1}, x_{2}\right),\left(x_{1}, x_{2}\right)$ et $\left(x_{2}, x_{3}\right)$ lorsque $R_{0,1}>1, R_{0,2}>1$ et $R_{0}>1$. Nous illustrons la convergence du sous-système (2.3) vers l'équilibre $E_{1}=(0.0275,0.0344,0.6394)$ lorsque $c_{1}=0.03, c_{2}=0.03$ et toutes les valeurs des autres paramètres sont dans le tableau 1 . Avec ces valeurs, nous avons $R_{0,1}=1.7280, R_{0,2}=1.6200$ et $R_{0}=3.3480$,
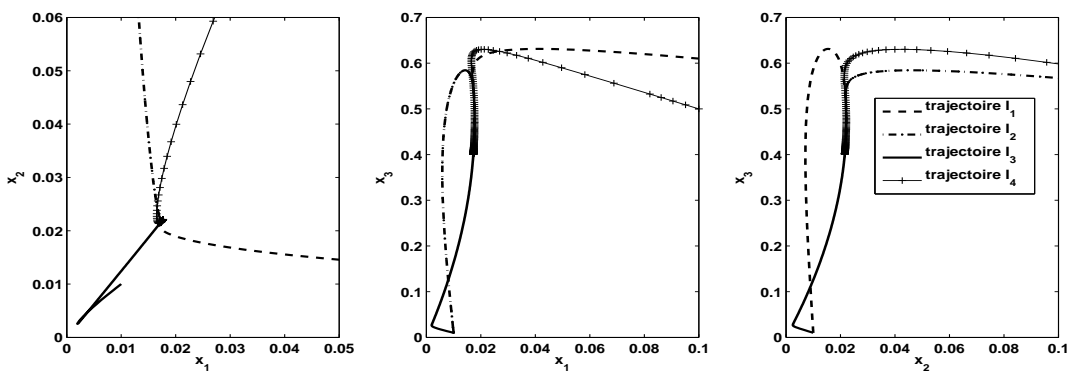

Figure 5 - Projections du portrait de phase du sous-système autonome (2.3) dans les plans $\left(x_{1}, x_{2}\right),\left(x_{1}, x_{2}\right)$ et $\left(x_{2}, x_{3}\right)$ lorsque $R_{0,1}<1, R_{0,2}<1$ et $R_{0}>1$. Nous illustrons la convergence du sous-système (2.3) vers l'équilibre $E_{1}=(0.0173,0.0216,0.4011)$ lorsque $c_{1}=0.016, c_{2}=0.016$ et toutes les valeurs des autres paramètres sont dans le tableau 1 . Avec ces valeurs, nous avons $R_{0,1}=0.9216, R_{0,2}=0.8640$ et $R_{0}=1.7856$.

Pour le sous-système autonome (2.4) lorsque $R_{0,2}>1$ nous avons montré théoriquement que $E_{0}$ est instable et $E_{2}$ est LAS (Corollaire 1). Mais, une simulation numérique montre que les trajectoires issues des quatre conditions initiales $I_{1}=(0.9,0.01,0.01)$, $I_{2}=(0.01,0.9,0.01), I_{3}=(0.01,0.01,0.01)$ et $I_{4}=(0.1,0.2,0.5)$ se trouvant aux coins du simplexe $\Omega$ et loin du voisinage de l'équilibre positive $E_{2}$ convergent toutes vers ce point lorsque $R_{0,2}>1$ (voir figure 6). 

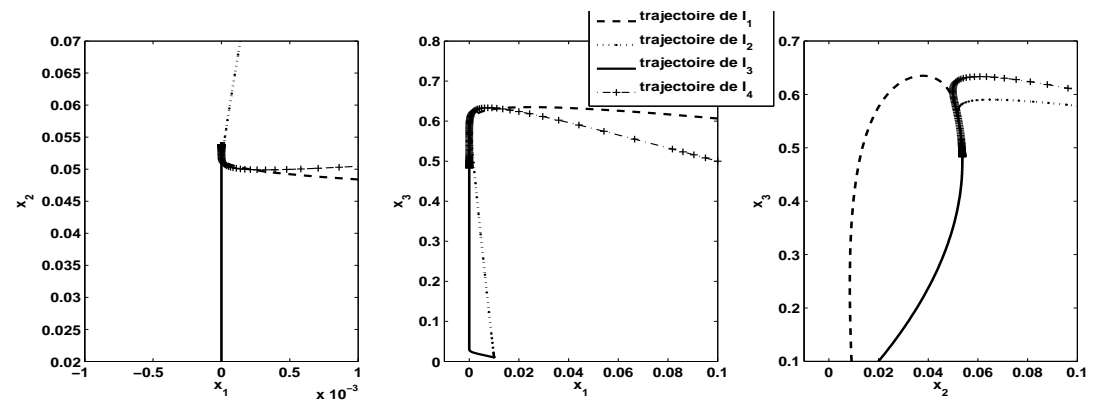

Figure 6 - Projections du portrait de phase du sous-système autonome (2.4) dans les plans $\left(x_{1}, x_{2}\right),\left(x_{1}, x_{2}\right)$ et $\left(x_{2}, x_{3}\right)$ lorsque $R_{0,2}>1$. Nous illustrons la convergence du sous-système (2.4) vers l'équilibre $E_{2}=(0,0.0537,0.4833)$ lorsque $c_{2}=0.04$ et toutes les valeurs des autres paramètres sont dans le tableau 1 . Avec ces valeurs, nous avons $R_{0,2}=2.1600$.

Les résultats numériques des figures $2,3,4,5$ suggèrent donc que pour certaines valeurs des paramtres telles que $R_{0}>1 E_{1}$ pourrait être globalement asymptotiquement stable pour le sous-système (2.3). De même, ceux de la figure 6 suggèrent que pour certaines valeurs des paramtres telles que $R_{0,2}>1 E_{2}$ pourrait être globalement asymptotiquement stable pour le sous-système (2.4) lorsque $R_{0,2}>1$

Toujours, lorsque $E_{0}$ est instable pour le sous-système (2.3) sous la condition $R_{0}>1$, si $R_{0,2} \leq 1$ alors $E_{0}$ est théoriquement GAS pour le sous-système (2.4). Dans ce cas, pour les valeurs particulières de $\alpha=\frac{1}{4}$ et $\alpha=\frac{2}{3}$, les simulations numériques montrent respectivement une convergence du système à commutation vers $E_{0}$ (voir figure 7) et vers un cycle limite (voir figure 8). 


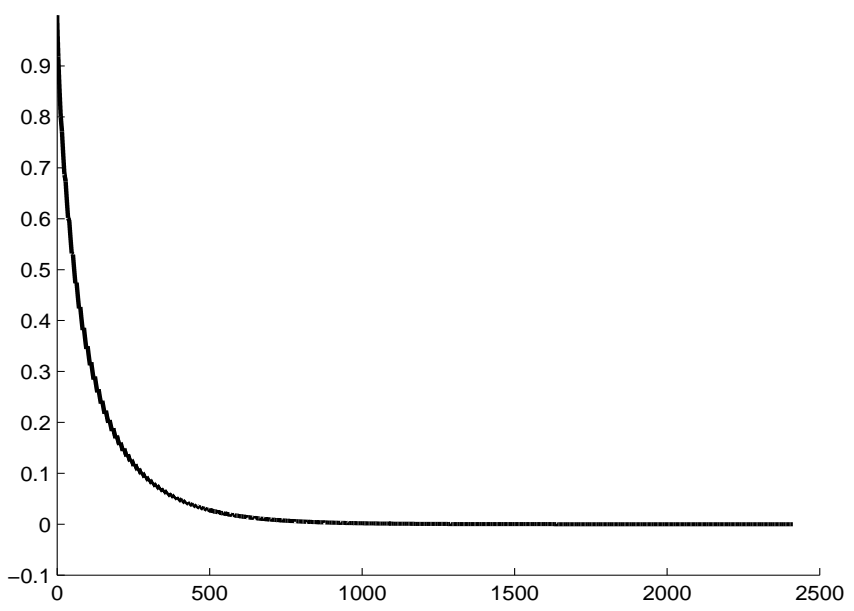

Figure 7 - Évolution au cours du temps de la population totale $y$ du système à commutation (2.2) avec la condition initiale $Y_{0}=(0.3,0.1,0.6) . c_{1}=0.03, c_{2}=0.01, T=12$, $\alpha=\frac{1}{4}$ et toutes les valeurs des autres paramètres sont dans le tableau 1 . Dans ce cas, nous avons $R_{0}=2.196>1, R_{0,1}=1,620$ et $R_{0,2}=0.576$. On a une convergence vers $E_{0}$. 


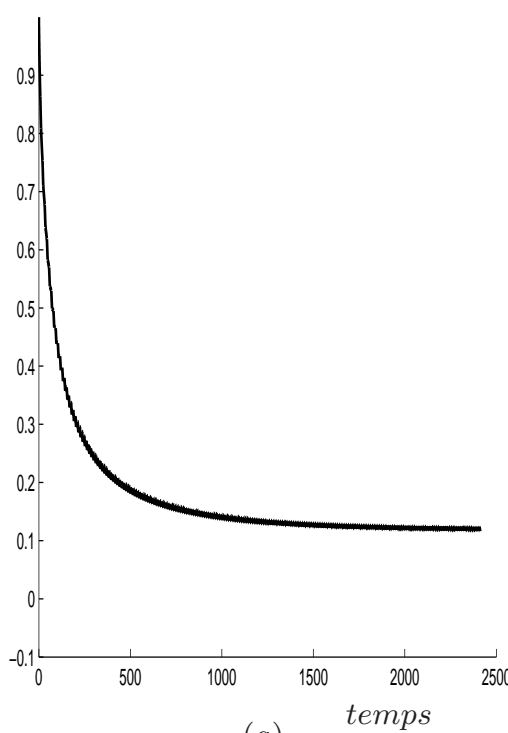

(a)

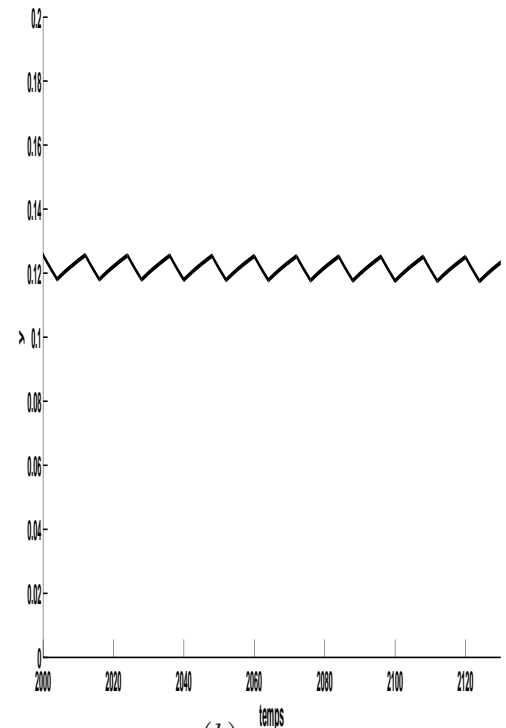

(b)

Figure 8 - (a) Évolution au cours du temps de la population totale du système à commutation (2.2) avec la condition initiale $Y_{0}=(0.3,0.1,0.6), c_{1}=0.03, c_{2}=0.01, T=12$, $\alpha=\frac{2}{3}$ et toutes les valeurs des autres paramètres sont dans le tableau 1 . Dans ce cas, nous avons $R_{0}=2.196>1, R_{0,1}=1,620$ et $R_{0,2}=0.576$. (b) est un zoom de la figure $(a)$ réalisé sur l'intervalle $[2000,2130]$ montrant la convergence vers un cycle limite.

Ainsi, ces deux figures ( 7 et 8 ) montrent que le paramètre $\alpha$ a une influence sur la dynamique du système à commutation. Certaines de ses valeurs engendrent la convergence du système vers $E_{0}$ ( voir figure 7 ) et d'autres vers un cycle limite ( voir figure 8). 


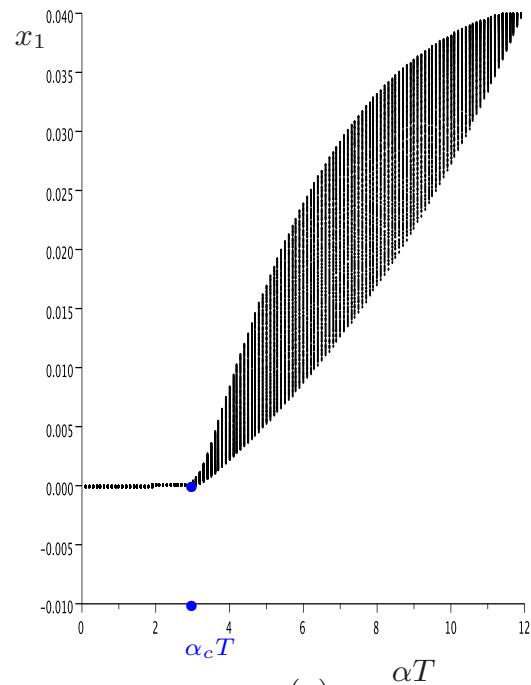

(a)

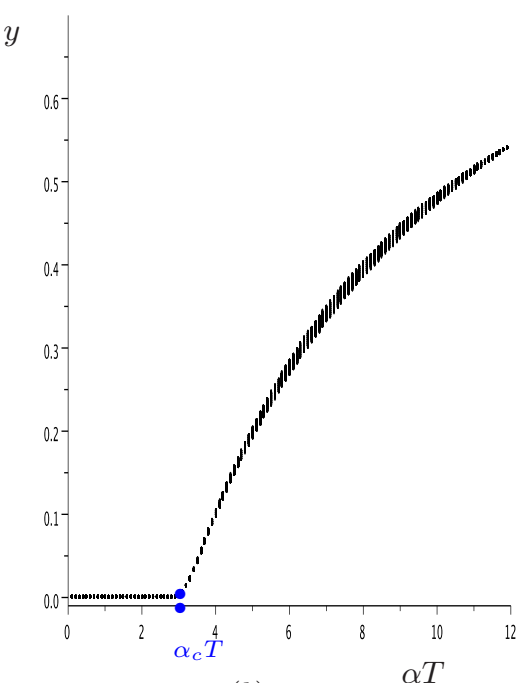

(b)

Figure 9 - Courbes des valeurs asymptotiques de $x_{1}$ figure $(a)$ et de la population totale $y$ figure (b) des solutions du système à commutation (2.2) en fonction de $\alpha T$ lorsque $c_{1}=0.03, c_{2}=0.01, T=12$ et toutes les valeurs des autres paramètres sont dans le tableau 1

Plus généralement, lorsque l'on trouve la solution numérique du système à commutation (2.2) sur une longue durée $d=1500$ mois et l'on représente en fonction de $\alpha T$ ou simplement de $\alpha$ la population totale $y$ ou $x_{1}$ aux 120 derniers temps (temps suffisamment grand pour voir le comportement asymptotique), alors l'on constate qu'il existe un paramètre de bifurcation $\alpha_{c}$ (voir figure 9). En effet, pour les valeurs de $\alpha$ inférieures à $\alpha_{c}$, $x_{1}$ et $y$ sont nulles aux 120 derniers temps : les solutions convergent vers l'équilibre nul. Mais, pour les valeurs de $\alpha$ supérieures à $\alpha_{c}$, la solution atteint un cycle limite puisque pour chacune de ces valeurs de $\alpha$, la variable $y$ (ou $x_{1}$ ) prend des valeurs distinctes aux 120 derniers temps et présentées par un trait vertical (voir figure 9).

\section{Conclusions}

Le Typha continue à proliférer dans différents pays du monde. Malheureusement, il n'existe pas à ce jour un moyen de lutte efficace contre cette plante. Néanmoins, le mo- 
dèle mathématique qui a été développé dans ce travail pourrait contribuer à une meilleure compréhension de la dynamique de population de la plante afin d'aborder au mieux son contrôle.

Dans ce papier, nous avons présenté un système différentiel non linéaire à commutation décrivant la dynamique de prolifération du Typha. Tout d'abord, nous avons étudié chacun de ses deux sous-systèmes. En celà, nous avons mis en évidence que l'existence et la stabilité de l'équilibre positif de chaque sous-système dépend du taux de reproduction de base ( $R_{0}$ ou $R_{0,2}$ ). Quand le taux de reproduction de base d'un sous-système est inférieur à 1 , l'étude de ce dernier montre une stabilité asymptotique de son équilibre $E_{0}$. Et quand son taux de reproduction de base est supérieur à 1 , nous avons une stabilité asymptotique de son équilibre positif. Ce paramètre rappelle le taux $\mathcal{R}_{0}$ des modèles démographiques où épidémiologiques, mais il faut noter que notre système n'est pas du type des modèles épidémiologiques auxquels la théorie générale s’applique [6].

Nous avons montré par des simulations numériques que le comportement du système à commutation est assez complexe. Précisément, pour certaines valeurs des paramètres telles que les deux sous-systèmes convergent vers $E_{0}$ lorsque $R_{0}<1$, le système à commutation converge vers $E_{0}$ quelque soit la séquence de commutation. Aussi, dans le cas où certaines valeurs des paramètres vérifient à la fois $R_{0}>1$ (le système associé à la présence de la reproduction sexuée converge vers $E_{1}$ ) et $R_{0,2}<1$ (le système associé à l'absence de la production sexuée converge vers $E_{0}$ ), le système à commutation (2.2) converge soit vers $E_{0}$, soit vers un cycle limite. Ces deux sénarios possibles montrent l'existence d'un paramètre seuil pour la durée de la période de reproduction sexuée $\alpha T$ au-delà duquel le système à commutation ne convergence plus vers $E_{0}$ mais vers un cycle limite pour un jeu de valeurs des paramètres tel que $R_{0}>1$.

On se propose dans la suite d'approfondir ces questions en utilisant la théorie des perturbations singulières, lorsque l'alternance entre la présence et l'absence de reproduction sexuée se fait très lentement $[3,8]$, et la théorie de la moyennisation, lorsque cette alternance est rapide [9].

Ainsi, l'étude théorique de ce modèle a permis de déterminer certains facteurs déterminants de la prolifération de la plante. Ce modèle pourrait être utiliser dans l'étude de stratégies de contrôle écohydrologique.

Remerciements : Les auteurs remercient, en dehors de leurs institutions, l'AUF (Horizons Francophones), l'UNESCO (C.B 32123110 DDK) et la coopération française (AIRES-Sud $n^{\circ} 7240$ ) qui ont financé leurs recherches. 


\section{A Calcul des points d'équilibre}

Les points d'équilibre du sous-système (2.3) vérifient

$$
\begin{aligned}
c_{1} x_{3}\left(1-x_{1}-x_{2}-x_{3}\right) & =\left(\gamma_{1}+\mu_{1}\right) x_{1} \\
c_{2} x_{3}\left(1-x_{1}-x_{2}-x_{3}\right) & =\left(\gamma_{2}+\mu_{2}\right) x_{2} \\
\gamma_{1} x_{1}+\gamma_{2} x_{2} & =\mu_{3} x_{3}
\end{aligned}
$$

Si une composante s'annule, alors $E_{0}=(0,0,0)$ est solution. Sinon $x_{1}^{*}>0, x_{2}^{*}>0$ et $x_{3}^{*}>0$ alors,

$$
1-x_{1}^{*}-x_{2}^{*}-x_{3}^{*} \neq 0 \text {. }
$$

puisque

$$
1-x_{1}^{*}-x_{2}^{*}-x_{3}^{*}=0 \text { implique } x_{1}=0 .
$$

En faisant le rapport membre à membre de (A.1) par (A.2) on obtient, après simplification

$$
\frac{c_{1}}{c_{2}}=\frac{\gamma_{1}+\mu_{1}}{\gamma_{2}+\mu_{2}} \frac{x_{1}^{*}}{x_{2}^{*}} .
$$

On en déduit l'expression suivante de $x_{2}^{*}$ en fonction de $x_{1}^{*}$

$$
x_{2}^{*}=\frac{c_{2}}{c_{1}} \frac{\gamma_{1}+\mu_{1}}{\gamma_{2}+\mu_{2}} x_{1}^{*}
$$

Or, l'équation (A.3) implique

$$
x_{3}^{*}=\frac{\gamma_{1}}{\mu_{3}} x_{1}^{*}+\frac{\gamma_{2}}{\mu_{3}} x_{2}^{*}
$$

Remplaçons dans (A.5) $x_{2}^{*}$ par sa valeur (A.4). On obtient l'expression suivante de $x_{3}^{*}$ en fonction de $x_{1}^{*}$

$$
x_{3}^{*}=\left[\frac{\gamma_{1}}{\mu_{3}}+\frac{\gamma_{2} c_{2}}{\mu_{3} c_{1}} \frac{\left(\gamma_{1}+\mu_{1}\right)}{\left(\gamma_{2}+\mu_{2}\right)}\right] x_{1}^{*} \text {. }
$$

Par conséquent,

$$
x_{3}^{*}=\frac{c_{1} \gamma_{1}\left(\gamma_{2}+\mu_{2}\right)+c_{2} \gamma_{2}\left(\gamma_{1}+\mu_{1}\right)}{\mu_{3} c_{1}\left(\gamma_{2}+\mu_{2}\right)} x_{1}^{*} .
$$

On peut écrire l'équation (A.1) sous la forme

$$
1-x_{1}^{*}-x_{2}^{*}-x_{3}^{*}=\frac{\gamma_{1}+\mu_{1}}{c_{1}} \frac{x_{1}^{*}}{x_{3}^{*}} .
$$


Remplaçons alors $x_{2}^{*}$ et $x_{3}^{*}$ par leurs valeurs (A.4) et (A.7) dans cette équation : on obtient, après simplification par $x_{1}^{*}$, l'équation suivante en l'unique variable $x_{1}^{*}$

$$
1-x_{1}^{*}-\frac{c_{2}}{c_{1}} \frac{\left(\gamma_{1}+\mu_{1}\right)}{\left(\gamma_{2}+\mu_{2}\right)} x_{1}^{*}-\frac{c_{1} \gamma_{1}\left(\gamma_{2}+\mu_{2}\right)+c_{2} \gamma_{2}\left(\gamma_{1}+\mu_{1}\right)}{\mu_{3} c_{1}\left(\gamma_{2}+\mu_{2}\right)} x_{1}^{*}=\frac{1}{R_{0}} .
$$

Cette équation s'écrit

$$
x_{1}^{*}\left[1+\frac{c_{2}}{c_{1}} \frac{\left(\gamma_{1}+\mu_{1}\right)}{\left(\gamma_{2}+\mu_{2}\right)}+\frac{c_{1} \gamma_{1}\left(\gamma_{2}+\mu_{2}\right)+c_{2} \gamma_{2}\left(\gamma_{1}+\mu_{1}\right)}{\mu_{3} c_{1}\left(\gamma_{2}+\mu_{2}\right)}\right]=1-\frac{1}{R_{0}} .
$$

On en déduit alors, en réduisant au même dénominateur

$$
x_{1}^{*} \frac{\mu_{3} c_{1}\left(\gamma_{2}+\mu_{2}\right)+\mu_{3} c_{2}\left(\gamma_{1}+\mu_{1}\right)+c_{1} \gamma_{1}\left(\gamma_{2}+\mu_{2}\right)+c_{2} \gamma_{2}\left(\gamma_{1}+\mu_{1}\right)}{\mu_{3} c_{1}\left(\gamma_{2}+\mu_{2}\right)}=1-\frac{1}{R_{0}} .
$$

Par conséquent,

$$
x_{1}^{*}=\frac{\mu_{3} c_{1}\left(\gamma_{2}+\mu_{2}\right)}{c_{1}\left(\gamma_{1}+\mu_{3}\right)\left(\gamma_{2}+\mu_{2}\right)+c_{2}\left(\gamma_{2}+\mu_{3}\right)\left(\gamma_{1}+\mu_{1}\right)} \frac{R_{0}-1}{R_{0}} .
$$

De l'équation (A.4) on déduit alors

$$
x_{2}^{*}=\frac{\mu_{3} c_{2}\left(\gamma_{1}+\mu_{1}\right)}{c_{1}\left(\gamma_{1}+\mu_{3}\right)\left(\gamma_{2}+\mu_{2}\right)+c_{2}\left(\gamma_{2}+\mu_{3}\right)\left(\gamma_{1}+\mu_{1}\right)} \frac{R_{0}-1}{R_{0}} .
$$

Et de l'équation (A.7), on déduit

$$
x_{3}^{*}=\frac{\mu_{3}\left(\gamma_{1}+\mu_{1}\right)\left(\gamma_{2}+\mu_{2}\right)}{c_{1}\left(\gamma_{1}+\mu_{3}\right)\left(\gamma_{2}+\mu_{2}\right)+c_{2}\left(\gamma_{2}+\mu_{3}\right)\left(\gamma_{1}+\mu_{1}\right)}\left(R_{0}-1\right) .
$$

Il ne reste plus qu'à montrer que $\left(x_{1}^{*}, x_{2}^{*}, x_{3}^{*}\right)^{t}$ appartient à $\Omega$.

$\left(x_{1}^{*}, x_{2}^{*}, x_{3}^{*}\right)^{t}$ appartient à $\Omega$. En effet, si $R_{0}>1$ nous avons $x_{1}^{*}>0, x_{2}^{*}>0$ et $x_{3}^{*}>0$.

De plus en remplaçant $x_{1}^{*}$ et $x_{3}^{*}$ dans le second membre de (A.8) on obtient l'expression

$$
1-x_{1}^{*}-x_{2}^{*}-x_{3}^{*}=\frac{1}{R_{0}}
$$

donc,

$$
x_{1}^{*}+x_{2}^{*}+x_{3}^{*}=1-\frac{1}{R_{0}} .
$$

Ainsi,

$$
x_{1}^{*}+x_{2}^{*}+x_{3}^{*}<1 \text {. }
$$


On en déduit que (3.3) est l'équilibre positif de (2.3).

Un point équilibre du sous-système (2.4) vérifie le système d'équations

$$
\left\{\begin{array}{ccc}
\left(\gamma_{1}+\mu_{1}\right) x_{1} & = \\
c_{2} x_{3}\left(1-x_{1}-x_{2}-x_{3}\right)-\left(\gamma_{2}+\mu_{2}\right) x_{2} & = & 0 \\
\gamma_{1} x_{1}+\gamma_{2} x_{2}-\mu_{3} x_{3} & = & 0
\end{array}\right.
$$

Donc, $x_{1}=0$ et on a

$$
\begin{aligned}
c_{2} x_{3}\left(1-x_{2}-x_{3}\right) & =\left(\gamma_{2}+\mu_{2}\right) x_{2} \\
\gamma_{2} x_{2} & =\mu_{3} x_{3}
\end{aligned}
$$

Si $x_{2}=0$ ou $x_{3}=0$, on obtient $E_{0}$ comme point d'équilibre.

Et par un raisonnement analogue, on obtient

$$
\begin{gathered}
x_{2}^{*}=\frac{\mu_{3}\left(R_{0,2}-1\right)}{R_{0,2}\left(\gamma_{2}+\mu_{3}\right)}, \\
x_{3}^{*}=\frac{\mu_{3}\left(\gamma_{2}+\mu_{2}\right)}{c_{2}\left(\gamma_{2}+\mu_{3}\right)}\left(R_{0,2}-1\right)
\end{gathered}
$$

On en déduit que (3.4) est l'équilibre positif de (2.4).

\section{B Bibliographie}

[1] J. G. ADAM « Présence de deux espèces de Typha dans la presqu'̂̂le du cap-Vert », Bulletin IFAN Dakar n ${ }^{\circ} 23$ (2), 1961.

[2] J. G. ADAM « Contribution à l'étude de la végétation du lac de Guiers (Sénégal) », Bulletin IFAN $\mathrm{n}^{\circ} 26(1): 1-72,1964$.

[3] H. Boudjellaba, T. SARI « Dynamic transcritical bifurcations in a class of slow-fast predator-prey models. », J. Differential Equations, $\mathrm{n}^{\circ}$ 6, 2205-2225, 246, 2009.

[4] C.-T. CHEN. «Linear System Theory and Design. », Holt, Rinehart and Winston, ${ }^{\circ}{ }^{\mathrm{N}}$ New York, 1984. pp. 417-4191

[5] FAYE V. M. « Etat actuel des peuplements de Typha domingensis Pers. Dans le delta du fleuve Sénégal et étude au laboratoire de la germination de la plante. », Mémoire de DEA. ISE, UCAD $\mathrm{n}^{\circ} 51 \mathrm{p}$.

[6] J.C. Kamgang, G. Sallet, « Computation of threshold conditions for epidemiological models and global stability of the disease-free equilibrium (DFE) », Math. Biosci, ${ }^{\circ}$ 1, 1-12, 2132008. 
[7] Khalil H. K. « Non linear Systems », Third Edition Prentice Hall, $\mathrm{n}^{\circ}$ 2002. ISBN 0-13-067389-7

[8] C. LOBRY, T. SARI, « The peaking phenomenon and singular perturbations. », ARIMA Rev. Afr. Rech. Inform. Math. Appl., n ${ }^{\circ}$ 487-516, 9, 2008.

[9] T. SARI, « Averaging for ordinary differential equations and functional differential equations. », The strength of nonstandard analysis, $\mathrm{n}^{\circ}$ 286-305, SpringerWienNewYork, Vienna, 2007.

[10] N. L. SARR, « Aspects socio-économiques de la prolifération de Typha domingensis dans le delta du fleuve Sénégal. », Mémoire de DEA, ISE, UCAD Dakar, n ${ }^{\circ} 49$ p, 2003.

[11] B. TreCA, «Les dégats aux semis de riz causés par les oiseaux d'eau dans le Delta du fleuve Sénégal. », Dakar. OSTOM, n 13p, 1989. 